\title{
Self-Referential Noise as a Fundamental Aspect of Reality *
}

\author{
Reginald T. Cahill and Christopher M. Klinger \\ Department of Physics, Flinders University ${ }^{\dagger}$ \\ GPO Box 2100, Adelaide 5001, Australia
}

\begin{abstract}
Noise is often used in the study of open systems, such as in classical Brownian motion and in Quantum Dynamics, to model the influence of the environment. However generalising results from Gödel and Chaitin in mathematics suggests that systems that are sufficiently rich that self-referencing is possible contain intrinsic randomness. We argue that this is relevant to modelling the universe, even though it is by definition a closed system. We show how a three-dimensional process-space may arise, as a Prigogine dissipative structure, from a nongeometric order-disorder model driven by, what is termed, self-referential noise.
\end{abstract}

\section{Introduction}

For over 300 years theoretical physics has very successfully modelled reality using geometrical models of the phenomena of space and time, and with deterministic fields and objects attached to the geometrical object which we call space. However there are indications from the quantum theory that there are processes, as in the Aspect experimental study [1] of the Einstein-PodolskyRosenfeld(EPR) effect (see Bell[2] for discussion), that a fundamental non-local random connectedness is needed to understand the quantum measurement process. For this and other reasons there have been attempts to construct more fundamental models of reality that do not begin with the assumption of an a priori geometry, and which are known as pregeometric models[3]. However, even extant pregeometric modellings of reality have had no success in explaining the phenomenon of space, and in particular why space is effectively three dimensional for most phenomena. As well the strong belief in the universality of the deterministic time evolution of physical systems (with the exception perhaps of the quantum measurement processes) does not take account of fundamental limitations that first began to appear with the discoveries of Gödel in mathematical logic.

\footnotetext{
${ }^{*}$ Contribution to the 2nd International Conference on Unsolved Problems of Noise, Adelaide 1999.

†E-mail: Reg.Cahill@flinders.edu.au, Chris.Klinger@flinders.edu.au
} 
Subsequent developments of Gödel's theorems by Chaitin [4 led to the discovery that mathematical systems sufficiently rich that self-referencing is possible contain intrinsic randomness. This appears to indicate a fundamental dichotomy between the limitations indicated by mathematic logic and the assumption of absolute determinism in theoretical physics. We argue that the resolution of this dichotomy is relevant to modelling the universe, and we show how a three-dimensional process-space may arise from a non-geometric order-disorder model as a Prigogine [5] dissipative structure driven by, what is termed, self-referential noise (SRN). We call this noise SRN to indicate its relationship to Gödel's theorems. However, note that this noise is not itself self-referential and nor is the model considered herein. Hence while noise is often used in the study of open systems, such as in classical Brownian motion and in Quantum Dynamics[6], to model the influence of the environment, here we argue that in the case of the universe, which by definition is a closed system, we must nevetheless use noise - not to take account of an environment, but to model the limitations indicated by logic [7, 8]. Patton and Wheeler [9] conjectured some time ago that Gödel's results in mathematics might be relevant to understanding cosmogony. For reasons discussed elsewhere[7] 8] we call this system a Heraclitean Process System (HPS).

\section{Process Systems}

Modelling reality at a fundamental level faces the problem of what to begin with? In [7, 8] we proposed a resolution to this problem by appealing to the phenomenon of self-organising criticality (SOC) [10]. In the proposed bootstrap model start-up components (called monads) acquire a selfconsistent meaning only as we reveal the fractal structures (i.e. criticality) that emerge. SOC systems have the property of universality, i.e. the behaviour of the system at a self-organised critical point is not uniquely characteristic of individual systems. Smolin 11 has discussed the possible relevance of SOC to cosmology.

The construction of a viable HPS can only be achieved at present by inspired guessing based in part upon the lessons of Quantum Field Theory (QFT)(see [12]) and the peculiarities of the quantum measurement process which indicate manifestations of non-linear and non-local random processes [6]. Our first HPS-SOC model is described by a non-linear noisy iterative map [7, \&], where the parameters $\alpha, \beta$ and $\gamma$ and the matrix $B_{c}$, see (2a), simplify the analysis:

$$
B_{i j} \rightarrow B_{i j}-\alpha\left(\beta^{-2} B+B^{-1}+\gamma B_{c}\right)_{i j}+w_{i j}, \quad i, j=1,2, \ldots, 2 M ; M \rightarrow \infty,
$$

We introduce, for convenience only, some terminology: we think of $B_{i j}$ as indicating the connectivity or relational strength between two monads $i$ and $j$ (these monads acquire a meaning later). The monads concept was introduced by Leibniz, who espoused the relational mode of thinking in response to and in contrast with Newton's absolute space. It is important to note that the iterations of the map do not constitute a priori the phenomenon of time, since they are to perform the function of producing the needed fractal structure which characterises universality in SOC. It is 
significant that this HPS is non-quantum, with the quantum phenomenon being emergent, see[7.

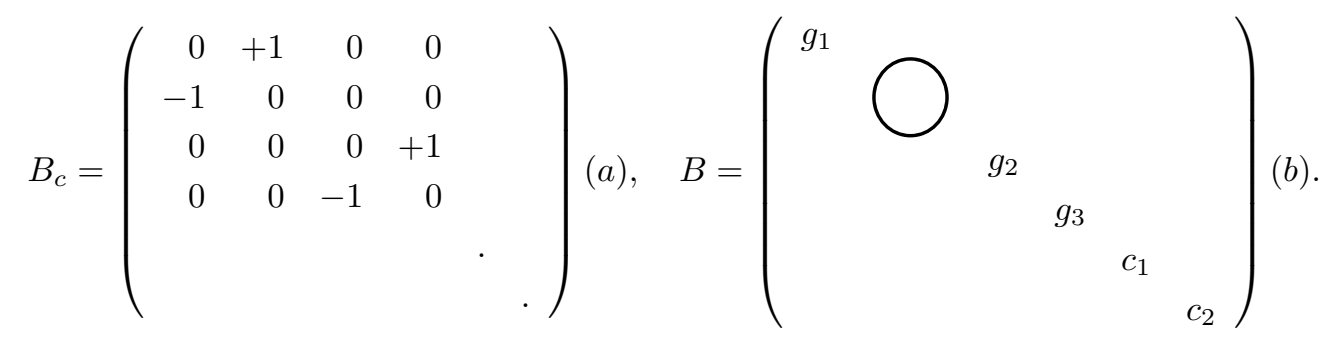

The monad $i$ has a pattern of dominant (larger valued $B_{i j}$ ) connections $B_{i 1}, B_{i 2}, \ldots$, where $B_{i j}=-B_{j i}$ avoids self-connection $\left(B_{i i}=0\right)$, and real number valued. The self-referential noise $w_{i j}=-w_{j i}$ are independent random variables for each $i j$ and for each iteration, and with variance $\eta$. Parameters satisfying $\alpha>\beta \gg \eta \gg \gamma$ result in identifiable emergent and evolving patterns. With the noise absent the iterator converges to the condensate $\beta B_{c}$ (but with $\gamma=0$ to one of $\beta R B_{c} R^{-1}$ where the matrix $R$ depends on the initial $B$ ). This behaviour is similar to the condensate of Cooper pairs in QFT[12], but here the condensate (indicating a non-zero dominant configuration) does not have any space-like structure. However in the presence of the noise, after an initial chaotic behaviour when starting the iterator from $B \approx 0$, the dominant mode is the formation of a randomised condensate $C \approx \mu \otimes B_{c}+B_{b}$, indicating $B_{c}$ but with the $\pm 1^{\prime} s$ replaced by $\pm \mu_{i}$ 's (where the $\mu_{i}$ are small and given by a computable iteration-dependent probability distribution $\mathcal{M}(\mu))$ and with a noisy background $B_{b}$ of very small $B_{i j}$.

The key discovery is that there is an extremely small (relative to $M$ ) self-organising process buried within this condensate and which has the form of a three-dimensional fractal process-space, which we now briefly explain. This structure is an example of a Prigogine far-from-equilibrium dissipative structure [5], emerging from the unstructured condensate and driven by the SRN. Under the mapping the noise term will produce rare large value $B_{i j}$, and these $B_{i j}$ will persist under the mapping (through more iterations than smaller valued $B_{i j}$ ) and form fluctuating patterns of connections, whose structure we now identify.

Consider the connectivity from the point of view of one monad, call it monad $i$. Monad $i$ is connected via these large $B_{i j}$ to a number of other monads, and the whole set of connected monads forms a tree-graph relationship. This is because the large links are very improbable, and a tree-graph relationship is much more probable than a similar graph involving the same monads but with additional links. The set of all large valued $B_{i j}$ then form tree-graphs disconnected from one-another; see Fig.1a. In any one tree-graph the natural 'distance' measure for any two monads within a graph is the smallest number of links connecting them. Let $D_{1}, D_{2}, \ldots, D_{L}$ be the number of nodes of distance $1,2, \ldots, L$ from monad $i$ (define $D_{0}=1$ for convenience), where $L$ is the largest distance from $i$ in a particular tree-graph, and let $N$ be the total number of nodes in the tree. See Fig.1b for an example.

Now consider the number $\mathcal{N}(D, N)$ of different $N$-node trees, with the same distance distribution $\left\{D_{k}\right\}$, to which $i$ can belong. By counting $\mathbb{Q}$ the different linkage patterns, together with 
permutations of the monads we obtain (3). We may compute the most likely tree-graph structure by maximising $\mathcal{N}(D, N)$ with respect to $\left\{D_{k}\right\}$. Fig.2 shows a typical result.

$$
\mathcal{N}(D, N)=\frac{(2 M-1) ! D_{1}^{D_{2}} D_{2}^{D_{3}} \ldots D_{L-1}^{D_{L}}}{(2 M-N) ! D_{1} ! D_{2} ! \ldots D_{L} !}
$$

Also shown is the approximate analytic form 13 $D_{k}=\frac{2 N}{L} \sin ^{2}(\pi k / L)$. These results imply that the most likely tree-graph structure to which a monad can belong has a distance distribution $\left\{D_{k}\right\}$ which indicates that the tree-graph is embeddable in a 3-dimensional hypersphere, $S^{3}$.
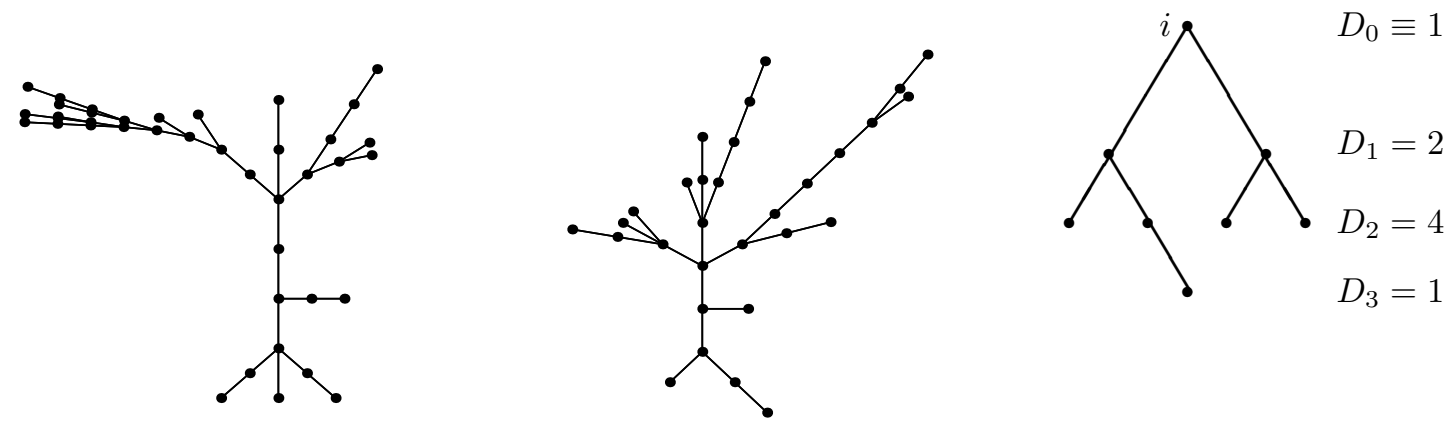

(a)

(b)

Figure 1: (a) Rare and large components of $B$ form disconnected tree-graphs, (b) An $N=8$ tree-graph with $L=3$ for monad $i$, with indicated distance distribution $D_{k}$.

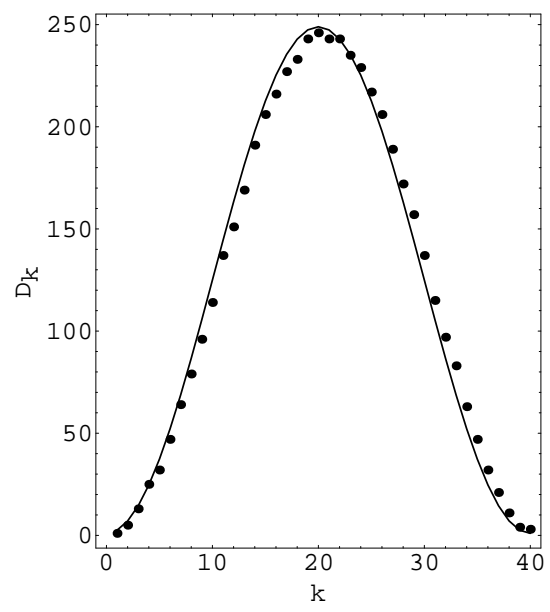

Figure 2: Points show the $D_{k}$ set and $L=40$ value found by numerically maximising (3) for fixed $N=5000$. Curve shows $D(k)=\frac{2 N}{L} \sin ^{d-1}\left(\frac{\pi k}{L}\right)$ with $d=3$ and $L=40$, showing excellent agreement, and indicating a weak embeddability in $S^{3}$.

We call these tree-graph $B$-sets gebits (geometrical bits). However $S^{3}$ embeddability of these gebits is a weaker result than demonstrating the necessary emergence of $S^{3}$-spaces, since extra 
cross-linking connections would be required for this to produce a strong embeddability; for evidence of this see [8].

The monads for which the $B_{i j}$ are, from the SRN term, large thus form disconnected gebits, and in (2b) we relabel the monads to bring these new gebits $g_{1}, g_{2}, g_{3}, .$. to block diagonal form, with the remainder indicating the small and growing thermalised condensate, $C=c_{1} \oplus c_{2} \oplus c_{3} \oplus \ldots$ In $2 \mathrm{~b}$ the $g_{i}$ indicate unconnected gebits, while the icon $\bigcirc$ represents older and connected gebits, and suggests a compact 3 -space (see below). The remaining very small $B_{m n}$, not shown in $(2 \mathrm{~b})$, are background noise only.

A key dynamical feature is that most gebit matrices $g$ have $\operatorname{det}(g)=0$, since most tree-graph connectivity matrices are degenerate[ [ These $\operatorname{det}(g)=0$ gebits form a reactive gebits subclass (i.e. in the presence of background noise $\left(g_{1} \oplus g_{2} \oplus g_{3} \oplus \text {.. }\right)^{-1}$ is well-defined and some elements large) of all those gebits generated by the SRN, and they are the building blocks of the dissipative structure. The self-assembly process is as follows: before the formation of the thermalised condensate $B^{-1}$ generates new connections (large $B_{i j}$ ) almost exclusively between gebits and the remaining nongebit sub-block (having det $\approx 0$ but because here all the involved $B_{i j} \approx 0$ ), resulting in the decay, without gebit interconnection, of each gebit. However once the condensate has formed (essentially once the system has 'cooled' sufficiently) the condensate $C=c_{1} \oplus c_{2} \oplus c_{3} \oplus \ldots$ acts as a quasi-stable (i.e. $\operatorname{det}(C)=\prod_{i} \operatorname{det}\left(c_{i}\right) \neq 0$ ) sub-block of $(2 \mathrm{~b})$ and the sub-block of gebits may be inverted separately. The gebits are then interconnected (with many gebits present cross-links are more probable than self-links) via new links formed by $B^{-1}$, resulting in the larger structure indicated by the $\bigcirc$ in (2b). Essentially, in the presence of the condensate, the gebits are sticky.

Continuing studies[7, 8] suggest that this network of self-assembling gebits forms a threedimensional fractal process-space (the $\bigcirc$ in $(2 \mathrm{~b})$ - essentially a Prigogine dissipative structure): fractal because sub-networks of gebits are themselves formed into larger networks. It is this rapidly expanding process-space that we associate with the phenomenon of space, and from the endophysics of this space the condensate is completely non-local. It is also clear, finally, that the original monads can be interpreted as themselves being networks of connected gebits. For this reason we thus have a bootstrap HPS[8] (i.e. the start-up components are identical in form to emergent components). After a transient regime of expansion, dominated by the interaction of topological defects produced during the early formation phase of the process-space, one would expect the processspace to undergo an exponential expansion because the growth in the number $n$ of gebits within the process-space would be described by a growth-decay equation

$$
\frac{d n}{d t}=a n-b n \text {. }
$$

This suggest that the HPS model may provide an explanation for the cosmological constant which now appears to be firmly established from observational evidence 14, 15.

A process-space is not equivalent to an inert geometrical space. In particular this implies a finite speed of propagation of any disturbance through the process-space and other distortion effects, caused by the need for the disturbance to be processed by the formation, interconnection 
and finally decay of the gebits. Toffoli[16] has speculated about such phenomena and its possible explanation of General Relativity-like effects within the area of Cellular Automata.

\section{Conclusions and Open Questions}

We have briefly discussed the problem which arises when we attempt to model and comprehend the universe as a closed system without assuming high level phenomena such as space and time. Our analysis is based upon the notion that a closed self-referential system, and the universe is ipso facto our only true instance, is necessarily noisy. This follows as a conjectured generalisation of the work of Gödel and Chaitin on self-referencing in the abstract and artificial game of mathematics. To explore the implications we have considered a non-quantum non-geometric non-linear noisy iterative map. The analysis of this map shows that the first self-organised structure to arise is a dynamical Prigogine-like dissipative process-3-space formed from interconnecting pieces of 3geometry - the gebits. We suggest that the concept of a non-local intrinsic noise has been a major missing component of traditional modelling of reality. As discussed elsewhere [7, 8] this model also generates the phenomenon of the present moment effect - an effect missing from the Newtonian and Einsteinian geometrical models, and an objectification process related to the phenomenon of (classical) objects and to the behaviour of quantum detectors.

Both analytical and numerical studies have indicated that the interconnecting gebits form a complex dynamical network once the system has cooled sufficiently for the non-local condensate to have formed. However a key open question is the proof, probably analytical, that this network is indeed three-dimensional. If this conjecture is confirmed then we would have for the first time

a model which predicts the emergence of the complex phenomenon of space, and one that is richer than that used in the present day geometric modelling in physics.

We thank Dr Derek Abbott and members of the UPoN99 Conference Secretariat for their efforts in organising this conference.

\section{References}

[1] A. Aspect, J. Dalibard and G. Roger, Phys. Rev. Lett. 49, 1804 (1982).

[2] J. Bell, Speakable and Unspeakable in Quantum Mechanics: Collected Papers on Quantum Mechanics, Cambridge Univ. Press 1989.

[3] J.A. Wheeler, Pregeometry: Motivations and Prospects, in Quantum Theory and Gravitation, A. R. Marlow, ed. (Academic Press, New York 1980).

[4] G.J. Chaitin, Information, Randomness and Incompleteness, 2nd ed. World Scientific, 1990.

[5] G. Nicholis and I. Prigogine, Self-Organization in Non-Equilibrium Systems: From Dissipative Structures to Order Through Fluctuations, J. Wiley \& Sons, New York, 1977. 
[6] I.C. Percival, Quantum State Diffusion, Cambridge Univ. Press, 1998.

[7] R.T. Cahill and C.M. Klinger, Bootstrap Universe from Self-Referential Noise, gr-qc/9708013.

[8] R.T. Cahill and C.M. Klinger, Self-Referential Noise and the Synthesis of Three-Dimensional Space, gr-qc/9812083, to be published in Gen. Rel. and Grav.

[9] C.M. Patton and J.A. Wheeler, Is Physics Legislated by Cosmogony?, in Quantum Gravity - an Oxford Symposium, p539, Eds.: C.J. Isham, R. Penrose and D.W. Sciama, Clarendon Press - Oxford 1975.

[10] P. Bak, C. Tang, and K. Wiesenfeld, Phys. Rev. Lett. 59, 381 (1987); Phys. Rev. A 38, 364(1988).

[11] L. Smolin, Cosmology as a Problem in Critical Phenomena, in the proceedings of the Guanajuato Conference on Complex Systems and Binary Networks, Springer, 1995, eds. R. Lopez-Pena, R. Capovilla, R. Garcia-Pelayo, H. Waalebroeck and F. Zertuche.

[12] R.T. Cahill and C.M. Klinger, Phys. Lett. A 223, 313 (1996).

[13] G. Nagels, Gen. Rel. and Grav. 17, 545 (1985).

[14] S. Perlmutter et al. Astrophys. J., in press (astro-ph/9812133).

[15] A. Riess, et al. Astron. J. 116, 1009(1998).

[16] T. Toffoli, in Complexity, Entropy and the Physics of Information, p 301, Ed.: W. H. Zurek, Addison-Wesley, 1990. 